\title{
Refining Patterns of Joint Hypermobility, Habitus, and Orthopedic Traits in Joint Hypermobility Syndrome and Ehlers-Danlos Syndrome, Hypermobility Type
}

\section{Silvia Morlino, ${ }^{1}$ Chiara Dordoni, ${ }^{2}$ Isabella Sperduti, ${ }^{3}$ Marina Venturini, ${ }^{4}$ Claudia Celletti, ${ }^{5}$ Filippo Camerota, ${ }^{5}$ Marina Colombi, ${ }^{2}$ and Marco Castori $^{1 *}$}

${ }^{1}$ Unit of Clinical Genetics, San Camillo-Forlanini General Hospital, Rome, Italy

${ }^{2}$ Division of Biology and Genetics, Department of Molecular and Translational Medicine, Medical Faculty, University of Brescia, Brescia, Italy

${ }^{3}$ Biostatistics, San Gallicano Dermatologic Institute, Rome, Italy

${ }^{4}$ Division of Dermatology, Department of Clinical and Experimental Sciences, Spedali Civili University Hospital,, Brescia, Italy

${ }^{5}$ Division of Physical Therapy and Rehabilitation, Department of Orthopedic Sciences, Sapienza University, Umberto I University Hospital, Rome, Italy

Manuscript Received: 26 April 2016; Manuscript Accepted: ? December 2016

Joint hypermobility syndrome (JHS) and Ehlers-Danlos syndrome, hypermobility type (EDS-HT) are two overlapping heritable disorders (JHS/EDS-HT) recognized by separated sets of diagnostic criteria and still lack a confirmatory test. This descriptive research was aimed at better characterizing the clinical phenotype of JHS/ EDS-HT with focus on available diagnostic criteria, and in order to propose novel features and assessment strategies. One hundred and eighty-nine (163 females, 26 males; age: $2-73$ years) patients from two Italian reference centers were investigated for Beighton score, range of motion in 21 additional joints, rate and sites of dislocations and sprains, recurrent soft-tissue injuries, tendon and muscle ruptures, body mass index, arm span/height ratio, wrist and thumb signs, and 12 additional orthopedic features. Rough rates were compared by age, sex, and handedness with a series of parametric and non-parametric tools. Multiple correspondence analysis was carried out for possible co-segregations of features. Beighton score and hypermobility at other joints were influenced by age at diagnosis. Rate and sites of joint instability complications did not vary according to age at diagnosis except for soft-tissue injuries. No major difference was registered by sex and dominant versus nondominant body side. At multiple correspondence analysis, selected features tend to co-segregate in a dichotomous distribution. Dolichostenomelia and arachnodactyly segregated independently. This study pointed out a more protean musculoskeletal phenotype than previously considered according to available diagnostic criteria for JHS/EDS-HT. Our findings corroborated the need for a re-thinking of JHS/EDS-HT on clinical grounds in order to find better therapeutic and research strategies. () 2017 Wiley Periodicals, Inc.

Key words: Beighton score; diagnostic criteria; dislocations; Ehlers-Danlos syndrome; joint hypermobility; Marfanoid habitus
How to Cite this Article:

Morlino S, Dordoni C, Sperduti I,

Venturini M, Celletti C, Camerota F, Colombi M, Castori M. 2017. Refining patterns of joint hypermobility, habitus, and orthopedic traits in joint hypermobility syndrome and Ehlers-Danlos syndrome, hypermobility type.

Am J Med Genet Part A 173A:914-929.

\section{INTRODUCTION}

Joint hypermobility syndrome (JHS) is a still neglected, heritable disorder coupling generalized joint hypermobility $(\mathrm{GJH})$ with variable consequences of joint instability, musculoskeletal pain,

Conflict of Interests: The authors declare that there is no conflict of interest concerning this work.

Grant sponsor: C.E.D.I. Onlus.

${ }^{*}$ Correspondence to:

Marco Castori, M.D., Ph.D., Division of Medical Genetics, San CamilloForlanini Hospital, Circonvallazione Gianicolense, 87, I-00152 Rome, Italy.

E-mail: m.castori@scf.gov.it

Article first published online in Wiley Online Library

(wileyonlinelibrary.com): 7 March 2017

DOI 10.1002/ajmg.a.38106 
and subtle skin features [Grahame et al., 2000]. Since the first report of $\mathrm{GJH}$ as a recognizable feature and the delineation of the Beighton score (BS [Kirk et al., 1967; Beighton et al., 1973]), our knowledge on the clinical impact of GJH is markedly changed. From the first attempt of defining GJH "benign" because it was originally thought associated with few consequences of a harmless body built or a physical asset, GJH is now increasingly re-interpreted as the "tip of the iceberg" of present or prospective systemic disorders. Among them, JHS is emerging as the most common condition, but its significant overlap with the apparently rarer Ehlers-Danlos syndrome hypermobility type (EDS-HT) inflames the scientific community [Castori and Colombi, 2015]. To date, many researchers and practitioners consider JHS and EDS-HT undistinguishable at the clinical level [Tinkle et al., 2009]. Although we are still waiting for the identification of the molecular basis of JHS and EDS-HT [De Paepe and Malfait, 2012; Mayer et al., 2013], there is increasing evidence for the co-segregation of JHS and EDS-HT as a single trait with variable expressivity in the familial cases (i.e., JHS/ EDS-HT) [Tinkle et al., 2009; Castori et al., 2014a].

Different sets of criteria are being used for diagnosing JHS (Brighton) and EDS-HT (Villefranche) [Beighton et al., 1998; Grahame et al., 2000]. Their overlap is notable as both include a high BS, pain, dislocations, and skin changes. However, differences exist concerning the definition of these criteria in the two sets. This probably reflects the different perspectives of the medical communities by whom they were originally delineated (i.e., rheumatologists for JHS, pediatricians and geneticists for EDS-HT) and the different populations in which these two diagnoses are more commonly ascertained (i.e., women for JHS and children for EDS-HT) [Tinkle et al., 2009]. In the last decade, the number of patients attending specialized clinics is increasing with an urgent need for standardizing diagnosis and management [Rombaut et al., 2015a; Palmer et al., 2016]. The original sets of criteria raised from an "opinion"-based approach from the converging experience of a few experts. Conversely, in the last years, many papers refine some of the previously identified pleiotropic features of the syndrome, such as the mucocutaneous and ocular involvement [Gharbiya et al., 2012; Castori et al., 2015], and underline other entirely novel manifestations, such as gastrointestinal involvement and autonomic dysfunction [Zarate et al., 2010; De Wandele et al., 2014]. The role of fatigue and chronic pain is also emphasized in JHS/EDS-HT [Voermans et al., 2010a,b; Voermans and Knoop, 2011]. While many articles offer a global impact of these features in JHS/EDS-HT, the observation of families and heterogeneous patients' cohorts suggest an age-influenced phenotype [Castori et al., 2010a, 2011, 2013, 2014a, 2015]. Nevertheless, though originally considered relevant features, little attention was put on the extent and evolution of anthropometry, consequences of joint instability and orthopedic traits in JHS/EDS-HT.

This is the first study systematically assessing anthropometry, orthopedic trait and distribution of whole-body joint hypermobility in JHS/EDS-HT. Data were extracted retrospectively from the medical records of 189 JHS/EDS-HT patients evaluated in two Italian expert centers for systemic hereditary connective tissue disorders. Our results could contribute to a better definition of the physical phenotype of JHS/EDS-HT and to refine its clinical diagnostic criteria for a prompt recognition of the disorder.

\section{PATIENTS AND METHODS}

This study was intended as a bi-centric, retrospective research gathering clinical data from two Italian centers for the diagnosis and management of systemic hereditary connective tissue disorders, particularly Ehlers-Danlos syndromes. The center with the highest number of patients lead the study and was considered the training center (TC). Data were then compared with the second center, which was named validation center (VC). All patients gave their informed consent to the study. This study was in accordance with the Helsinki Declaration of 1975, as revised in 1983.

\section{Patients' Selection and Phenotype Definition}

Patients of the TC were selected from the jointed service for joint hypermobility and Ehlers-Danlos syndrome at the Medical Genetics Outpatient Service of the San Camillo-Forlanini Hospital and the Physical Medicine and Rehabilitation Center of the Policlinico Umberto I University Hospital in Rome. Patients of the VC were enrolled from the Center for the Diagnosis and Management of Ehlers-Danlos syndromes and Hereditary Connective Tissue Disorders at the University of Brescia and Spedali Civili University Hospital in Brescia. All patients underwent a direct clinical evaluation aimed at extensively defining the phenotype and associated management issues. The general assessment followed standard procedures as previously described [Castori et al., 2014a, 2015]. In brief, all patients were evaluated for the available clinical diagnostic criteria for JHS (Brighton criteria) [Grahame et al., 2000] and Ehlers-Danlos syndrome (Villefranche criteria) [Beighton et al., 1998]. When necessary, other sets of criteria for cognate disorders, such as the revised Ghent criteria for Marfan syndrome [Loeys et al., 2010] were also applied. Differential diagnosis included heart ultrasound and ophthalmological survey and, when necessary, bone densitometry and spine and brain MRI, as well as a non-invasive assessment of the vascular tree, according to the recently published Italian diagnostic approach for Ehlers-Danlos syndromes [Castori and Colombi, 2016]. In case of persistent doubts concerning the overlap with other hereditary connective tissue disorders (in particular, vascular and classical EDS, and Loeys-Dietz syndromes), appropriate molecular studies were performed by the national reference laboratory for the molecular diagnosis of EDS and related conditions (Brescia). However, supplementary molecular studies were carried out in a few patients and most cases were limited to the exclusion of point mutations and intragenic rearrangements of COL5A1 and COL5A2 as previously stated [Ritelli et al., 2013]. Once excluded partially overlapping hereditary soft connective tissue disorders on clinical grounds and, if considered necessary, by molecular tools, all patients were classified as affected by JHS (Brighton criteria only), EDS-HT (Villefranche criteria for EDS-HT only) or JHS + EDS-HT (Brighton and Villefranche criteria), as previously detailed [Castori et al., 2014a]. Both sets of criteria were strictly applied. The clinical diagnosis of EDS-HT was attributed only in presence of both major criteria, while that of JHS was fixed in presence of both major, one major and two minor, or four minor 
criteria [Beighton et al., 1998; Grahame et al., 2000]. As EDS-HT is considered the unique major Ehlers-Danlos syndrome subtype lacking a confirmatory laboratory test [Mayer et al., 2013], diagnosis remained clinical in all patients.

\section{Patients' Evaluation}

Age at examination, sex and handedness was registered in all available subjects. GJH was primarily assessed by the BS [Beighton et al., 1973]. This score is composed of five maneuvers: four of them are tested passively on both sides of the body and one is tested actively. BS is a 9-point evaluation with attribution of one point in the presence of any of the following: (a) passive apposition of the thumb to the flexor aspect of the forearm (one point for each hand), (b) passive dorsiflexion of the $\mathrm{V}$ finger beyond $90^{\circ}$ (one point for each hand), (c) hyperextension of the elbow beyond $10^{\circ}$ (one point for each arm), (d) hyperextension of the knees beyond $10^{\circ}$ (one point for each leg), (e) forward flexion of the trunk with the knees extended and the palms resting flat on the floor. In non-collaborative subjects (such as, toddlers and adults in wheelchair), the upper end of the sum was reduced by one point by excluding the maneuver for forward flexion of the trunk. In this case, the highest score was $8 / 8$. The 5-point questionnaire was also used as a pre-screening tool [Hakim and Grahame, 2003], but their results were not further used for the present work as this tool was not yet included in any set of diagnostic criteria. Range of motion (ROM) was also assessed for a range of joints and group of joints not included in the BS. As well representative of the entire human body, we selected temporomandibular joint (TMJ) (hypermobile for a full opening of $>50 \mathrm{~mm}$ ), 2nd-4th metacarpophalangeal (MCP) joints (hypermobile with a dorsal passive extension $>40^{\circ}$ ), proximal and distal phalangophalangeal joints at 2nd-4th fingers (hypermobile with a dorsal active extension $>0^{\circ}$ ), metatarsophalangeal (MTP) joint at 1st toe (hypermobile with a dorsal passive extension $>70^{\circ}$ ), hip (hypermobile with an abduction with leg extended $>45^{\circ}$ ), ankle (hypermobile with a passive dorsiflexion $>30^{\circ}$ ), neck in rotation (hypermobile for an active rotation $>90^{\circ}$ at each side), and lateral flexion (hypermobile for an active lateral flexion $>45^{\circ}$ at each side), thoracolumbar spine in rotation (hypermobile for a passive rotation $>30-45^{\circ}$ at each side) and lateral flexion (hypermobile for an active lateral flexion $>35^{\circ}$ at each side). All standard values for these extra joints were extracted and only slightly adapted from Clarkson [2012] and obtained with the use of an orthopedic goniometer or a flexible tape. Presence of hypermobility (interpreted as a dichotomous feature: yes or not) was registered on both sides.

A set of static orthopedic features were systematically registered at direct examination and included: (fixed) subluxation of the distal ulna, (fixed) subluxation of the first metacarpal bone, cubitus valgus, pectus excavatum, pectus carinatum, dorsal hyperkyphosis (clinical examination), lumbar hyperlordosis (clinical examination), scoliosis (always of mild degree in JHS/EDS-HT $\leq 20^{\circ}$ at clinical/radiological examination), genuum valgus, hallux valgus, and in-toeing gait/metatarsus varus. History annotation comprised the site and number of past luxations/ subluxations and sprains. Presence of (recurrent) soft-tissue injuries was registered also in presence of three or more episodes. Single or multiple events of tendon and muscle ruptures were also registered. Standard biometry included height $(\mathrm{cm})$, weight $(\mathrm{kg})$, and arm span $(\mathrm{cm})$, from which the body-mass index (BMI) and arm span/height ratio were calculated. As applied in routine clinical examination, in patients $>15$ years of age, the presence of leptosomic/ectomorphic and picnic/endomorphic built was established with a BMI $<19$ and $>25$, respectively in both sexes. "Dolichostenomelia" was established with an arm span/height ratio $>1.03$ according to the Brighton criteria [Grahame et al., 2000]. Complementarily, an arm span/height ratio $<0.97$ was arbitrarily considered indicative for "brachymelia." The presence of wrist and thumb (Steinberg) sign (monolateral or bilateral) was also documented. Data on upper/lower body segment ratio were not available in both groups.

Data from the VC were limited compared to TC and did not include joint mobility measurement outside the BS and selected anthropometric and orthopedic features. ROM was measured with an orthopedic goniometer in the TC only, while the BS was established subjectively. Such a difference was originally established by the senior investigators of the two centers as a result of partially divergent assessment approaches, still in according to available diagnostic criteria. Indeed, both the Vilfefranche and Brighton criteria do not explain how to measure ROT for BS score calculation. Recently, a worldwide lack of consensus in the assessment of joint mobility and the diagnosis of JHS/EDS-HT was highlighted by a questionnaire study among European and American experts [Remvig et al., 2014].

\section{Statistical Analysis}

A series of descriptive statistics were used to summarize pertinent study information. Chi-square or Fisher's exact test was performed for the comparison of categorical variables. The linear regression model was used to investigate possible relationship between continuous variables. Comparison between the presence/absence of selected features and continuous variables (i.e., age at examination and BS) was performed using the Mann-Whitney non parametric test. All $P$ values were reported as 2 -sided and $P$ values $<0.01$ denotes statistically significant association. Multivariate logistic regression analysis considering age, gender, and center was carried out to examine the associations for each feature/or group of features being studied. All analyses were performed by using the SPSS software (SPSS version 21.0, SPSS Inc., Chicago, IL).

Phenotype and evolution of JHS/EDS-HT are extremely variable [Castori, 2012; Castori et al., 2013]. The reasons for such a variability are still obscure, but genetic heterogeneity may be a major contributor. This could also explain as to why the molecular basis of JHS/EDS-HT remains unraveled. The identification of discrete sub-phenotypes seems a good approach to ease the future molecular characterization of JHS/EDS-HT and/or to identify clues for predicting developmental trajectories. For this reason, we further explored our data searching for possible clusters of discrete features within our patients' sample. Multiple correspondence analysis (MCA), which is a descriptive technique designed to analyze simple 2-way and multi-way tables, was considered an adequate tool to evaluate the possible relationship among selected variables and identify specific profiles. Associations between features are represented graphically in the MCA, which is a graphic representation of the statistical relationships between distinct features, whose position in the graphic is exclusively informative 
TABLE I. Descriptive Features of the Two Study Groups-Joint Hypermobility and Joint Instability Complications

\section{Feature}

No. of patients

Females

Males

Total

Age (years]

Median

Range

Total

Beighton score

Median

Range

Total

Joint hypermobility, Beighton score

1st finger right

1st finger left

5 th finger right

5 th finger left

Elbow right

Elbow left

Knee right

Knee left

Spine

Total

Joint hypermobility, other sites

TMJ

2nd-4th MCPs right

2nd-4th MCPs left

2nd-4th PIPs right

2nd-4th PIPs left

2nd-4th DIPs right

2nd-4th DIPs left

MTPs right

MTPs left

Ankle right

Ankle left

Hip right

Hip left

Neck rotation right

Neck rotation left

Neck flexion right

Neck flexion left

Full spine rotation right

Full spine rotation left

Full spine flexion right

Full spine rotation left

Total

Dislocations

1-2

$3-10$

$>10$

Habitual/recurrent

Fingers

Wrists

Elbows

Shoulders

Heels

Knees/rotulae

\section{Training Center [\%]}

$106(85.5)$
$18(14.5)$
$124(100)$

32

2-73

124

6.00

0-9

124

85 (68.5)

93 (75.0)

87 (70.2)

96 (77.4)

42 (33.9)

60 (48.4)

71 (57.3)

73 (58.9)

51 (41.5)

124

46 (37.1)

84 (67.7)

87 (70.2)

40 (32.3)

50 (40.3)

58 (46.8)

61 (49.2)

51 (43.6)

52 (44.4)

32 (27.4)

32 (27.4)

49 (41.9)

49 (41.9)

38 (32.5)

35 (29.9)

57 (48.7)

56 (47.9)

78 (66.7)

78 (66.7)

42 (35.9)

44 (37.6)

118-124

13 (10.5)

21 (16.9)

42 (33.9)

8 (6.5)

9 (7.3)

9 (7.3)

9 (7.3)

38 (30.6)

11 (8.9)

22 (17.7)

\section{Validation Center [\%]}

$$
\begin{array}{r}
57 \text { (87.7) } \\
8(12.3) \\
65(100)
\end{array}
$$

$$
\begin{gathered}
37 \\
5-70 \\
65 \\
\\
4.00 \\
0-9 \\
65
\end{gathered}
$$

28 (43.1)

31 (47.7)

16 (24.6)

$26(40.0)$

38 (58.5)

35 (53.8)

18 (27.7)

22 (33.8)

37 (56.9)

65

NA

NA

NA

NA

NA

NA

NA

NA

NA

NA

NA

NA

NA

NA

NA

NA

NA

NA

NA

NA

NA

7 (10.8)

13 (20.0)

18 (27.7)

10 (15.4)

4 (6.2)

7 (10.8)

5 (7.7)

33 (50.8)

9 (13.8)

13 (20.0)
P-Value

$0.259^{1}$

$<0.0001^{1}$

0.001

$<0.0001$

$<0.0001$

$<0.0001$

0.002

0.48

$<0.0001$

0.001

0.04

NA

NA

NA

NA

NA

NA

NA

NA

NA

NA

NA

NA

NA

NA

NA

NA

NA

NA

NA

NA

NA

0.95

0.6

0.39

0.05

1.0

0.41

1.0

0.007

0.29

0.7

(Continued) 


\begin{tabular}{|c|c|c|c|}
\hline $\begin{array}{l}\text { Feature } \\
\text { Hips }\end{array}$ & $\begin{array}{c}\text { Training Center [\%] } \\
31(25.0)\end{array}$ & $\begin{array}{c}\text { Validation Center [\%] } \\
19(29.2]\end{array}$ & $\begin{array}{l}P \text {-Value } \\
0.53\end{array}$ \\
\hline TMJ & $32(25.8)$ & $28(43.1)$ & 0.01 \\
\hline Vertebrae & $3(2.4)$ & $2(3.1)$ & 0.79 \\
\hline Ribs & $1(0.8)$ & $1(1.5)$ & 1.0 \\
\hline Total & 124 & 65 & \\
\hline \multicolumn{4}{|l|}{ Major sprains } \\
\hline $1-2$ & 10 (8.1) & $10(15.4)$ & 0.12 \\
\hline $3-10$ & 18 (14.5] & $13(20.0)$ & 0.33 \\
\hline$>10$ & $47(37.9)$ & 21 (32.3) & 0.45 \\
\hline Habitual/recurrent & $5(4.0)$ & 6 [9.2] & 0.19 \\
\hline Fingers/wrists & 12 (9.7) & $6(9.2)$ & 0.92 \\
\hline Elbows & $1(0.6)$ & $1(1.5)$ & 1.0 \\
\hline Heels & 75 (60.5) & 38 [58.5] & 0.79 \\
\hline Knees & $15(12.1)$ & $17(26.2)$ & 0.01 \\
\hline Total & 124 & 65 & \\
\hline \multicolumn{4}{|c|}{3 or more soft tissue injuries } \\
\hline Feature & $46(37.1)$ & $26(40.0)$ & 0.7 \\
\hline Total & 124 & 65 & \\
\hline \multicolumn{4}{|l|}{ Tendon ruptures } \\
\hline Feature & $4(3.2)$ & $3(4.6)$ & 0.69 \\
\hline Total & 124 & 65 & \\
\hline \multicolumn{4}{|l|}{ Muscle ruptures } \\
\hline Feature & $5(4.1)$ & $1(1.5)$ & 0.67 \\
\hline Total & 122 & 65 & \\
\hline
\end{tabular}

[Greenacre, 1984; Lebart et al., 1984]. Features to be analyzed were selected arbitrarily among those which can be easily interpreted as dichotomic and are more representative of what is to date considered the JHS/EDS-HT "core phenotype."

\section{RESULTS}

The TC and VC included 124 and 65 patients, respectively, for a total of 189 individuals from 2 to 73 years of age. Tables I and II summarized rough data obtained in the TC and VC. Groups were comparable for age and sex bias, with the well-known skewed sex ratio due to an excess of affected females (Table I [Castori et al., 2010b]). Significant differences were noted concerning the BS range with a median score of six and four in the TC and VC, respectively. Information on joint instability complications (i.e., dislocations, sprains, soft-tissue injuries, and ruptures) were comparable between groups with a few exceptions, including the number of dislocations at shoulders which were more common in the VC (Table I). Anthropometry and the rate of selected orthopedic traits did not differ between groups (Table II).

\section{Beighton Score [and Age]}

In previous ecologic studies, an inverse correlation between BS and age was suggested for Italian patients [Castori et al., 2011, 2014a]. In the present work, correlation between BS and age at diagnosis was further investigated and a statistically significant inverse relationship was confirmed in both groups, with a linear $\mathrm{R}$ square of 0.33 ( $P$-value: $<0.0001)$ and 0.17 ( $P$-value: 0.001$)$ in the TC and VC, respectively (Fig. 1A). This phenomenon was comparable between the two groups $(P=0.38)$.

In a pilot research on $50 \mathrm{JHS} / \mathrm{EDS}-\mathrm{HT}$ Italian patients, we found a cut-off of 33 years for the transition from a positive to a negative BS [Castori et al., 2011]. In the present study, the same trend was found in the TC group with a $P$-value of $<0.0001$, while borderline $P$ values were found in the VC (i.e., 0.02; Fig. 1B). Still in according with our previous observations [Castori et al., 2011], we evaluated the presence/absence of the single items of the BS by age at examination (Table III). Results were different between groups. In the TC, the mean age was significantly lower among individuals with hypermobility at any single joint compared to those without, except for the right knee and spine. In the $\mathrm{VC}$, all results were comparable between the two groups (i.e., presence and absence).

\section{Hypermobility in Other Joints (and Age)}

In the $\mathrm{TC}$, we also evaluated ROM in 21 additional joints or groups of joints outside the five maneuvers of the BS (Table I). Figure 2 compares age at examination and presence/absence (dichotomic trait) of joint hypermobility (i.e., ROM values above normal) for each of these 21 extra joints. Statistically significant values were obtained for all sites, except TMJ, MTP joints (bilateral), ankles (bilateral), and spine (rotation). In these joints, median age, and age range of patients showing (presence) and not showing (absence) hypermobility were comparable, while the median age of 


\section{TABLE II. Descriptive Features of the Two Study Groups-Anthropometry, Habitus and Orthopedic Traits}

\section{Feature}

BMI

Median

Range

Total

Arm span/height ratio

Median

Range

Total

Habitus (BMI)

Leptosomic

Picnic

Total

Habitus (arm span)

Dolichostenomelia

Brachymelia

Total

Wrist sign

Right

Left

Monolateral

Bilateral

Total

Thumb sign

Right

Left

Monolateral

Bilateral

Total

Orthopedic traits

FDSDU right

FDSDU left

1st MCP subluxation right

1st MCP subluxation left

Pectus carinatum

Pectus excavatum

Dorsal hyperkyphosis

Lumbar hyperlordosis

Scoliosis $(<20$ degrees $)$

Cubitus valgus right

Cubitus valgus left

Genuum valgus right

Genuum valgus left

Flexible flatfoot right

Flexible flatfoot left

In-toeing gait right

In-toeing gait left

Hallux valgus right

Hallux valgus left

Total
Training Center [\%]

22.25

16.16-35.16

96

1.045

0.91-1.073

121

15 (15.6)

$23(24.0)$

96 (100)

30 (24.8)

12 (9.9)

121

26 (21.5)

27 (22.3)

5 (4.1)

24 (19.8)

121

11 (9.0)

14 (11.5)

$5(4.1)$

10 (8.2)

121

81 (65.9)

86 (69.4)

7 (5.6)

5 (4.0)

5 (4.2)

$9(7.5)$

$83(67.5)$

$86(69.4)$

67 (54.0)

65 (52.4)

52 (41.9)

61 (49.2)

64 (51.6)

72 (58.1)

67 (54.0)

$10(8.2)$

$10(8.2)$

33 (27.0)

35 (28.7)

120-124
Validation Center [\%]

21.57

15.24-36.33

57

NA

$10(17.5)$

13 (22.8)

57 (100)

NA

9 (15.8)

$10(17.5)$

7 (12.3)

5 (8.8)

57

$4(7.0)$

$5(8.8)$

$3(5.3)$

3 (5.3)

57

NA

NA

NA

NA

1 (1.5)

9 (13.8)

$40(61.5)$

44 (67.7)

$39(60.0)$

34 (52.3)

28 (43.1)

22 (33.8)

24 (36.9)

33 (50.8)

32 (49.2)

7 (10.8)

6 (9.2)

26 (40.0)

28 (43.1)

65
NA

NA

$P$-Value

$0.49^{1}$

0.76

0.87

0.37

0.46

0.06

0.06

0.78

0.58

0.71

0.76

NA

NA

NA

NA

0.33

0.16

0.41

0.82

0.43

0.99

0.88

0.04

0.05

0.34

0.53

0.56

0.81

0.07

0.05

BMI, body mass index; FDSDU, fixed dorsal subluxation of the distal ulna; MCP, metacarpophalangeal (joint); NA, not available. ${ }^{1}$ Mann-Whitney U test. 


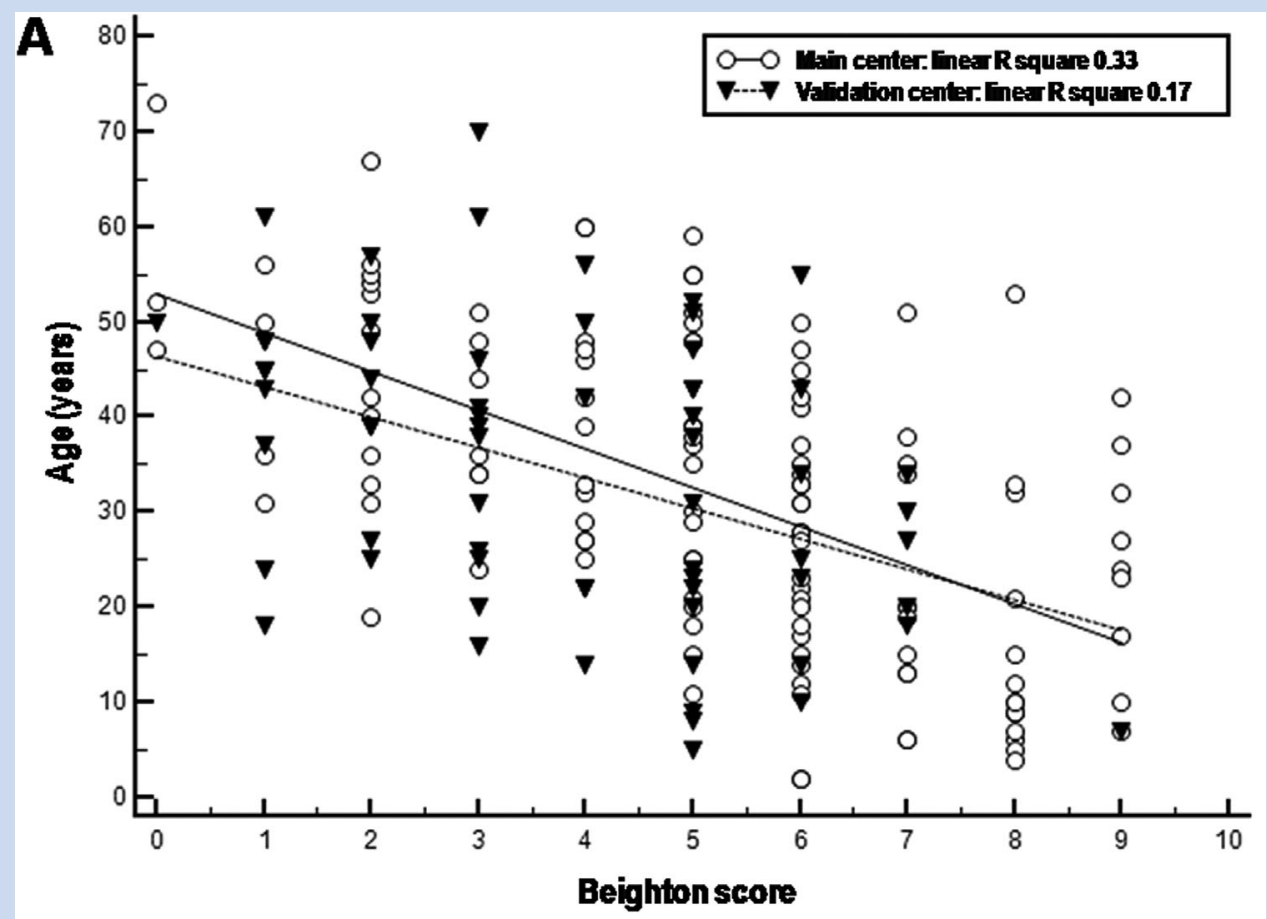

B

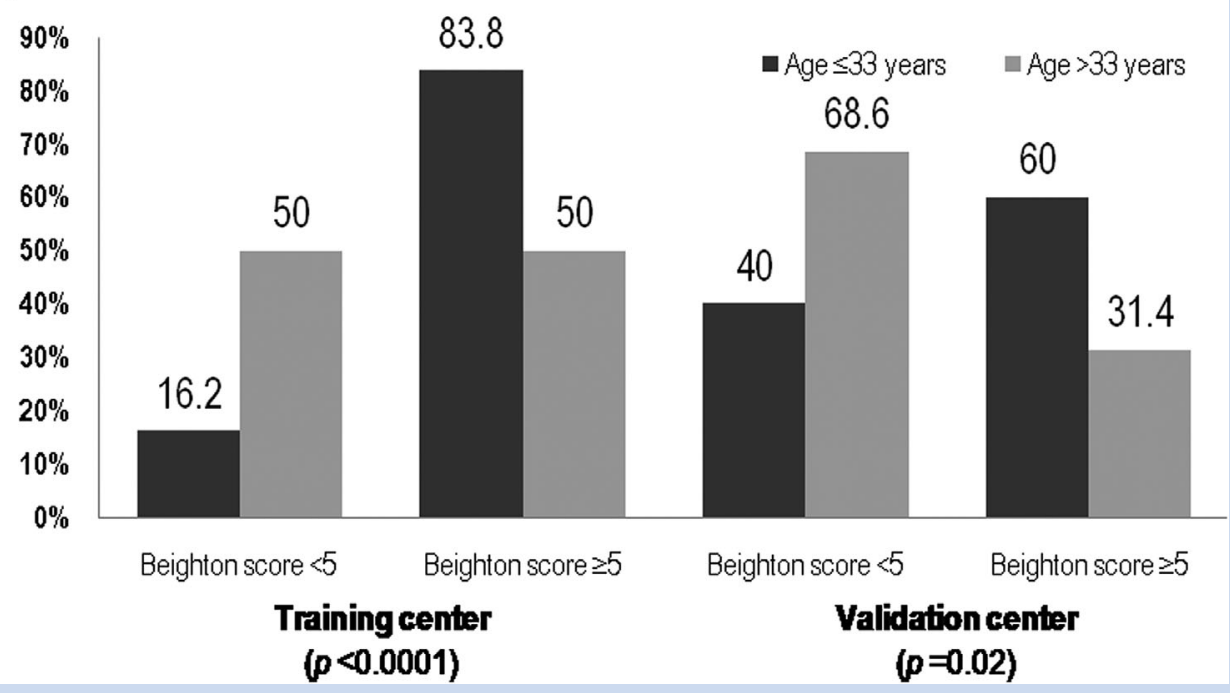

FIG. 1. (A) Scatter plot showing inverse linear correlation between age at examination and Beighton score in the training center (white circles] and validation center (inverse black triangles]. In both cohorts an inverse correlation between Beighton score and age was demonstrated. The $P$-value of the R square was $<0.0001$ and 0.001 in TC and VC, respectively. (B) Distribution of patients in both groups with or without a positive Beighton score in relation to their age. A dichotomous distribution with a cut-off of 33 years was used according to previous study.

patients presenting the feature was significantly lower than those without in all other joints. We also tried to use these 21 extra sites of joint hypermobility as a tool complementary to BS. We arbitrarily attributed one point to the presence and 0 point to the absence of hypermobility in each joint (or group of joints), similarly to the five maneuvers of the BS. The sum ranged from 0 (i.e., absence of all extra hypermobile joints) to 21 (i.e., presence of all extra hypermobile joints). Comparably to the BS (Fig. 1A), the overall score diminished according to age at evaluation with a linear $\mathrm{R}$ square of 0.33 also for these extra joints (Fig. 3).

\section{Physical Examination and Sex}

Differences between sexes concerning somatic manifestations were minimal. Concerning the BS, an excess of hypermobile spine ( $P$-value: 0.007$)$ were noted in women, while borderline $P$ values 


\begin{tabular}{|c|c|c|c|c|c|c|}
\hline \multirow{3}{*}{$\begin{array}{l}\text { Beighton score item } \\
\text { 1st finger right }\end{array}$} & \multicolumn{3}{|c|}{ Training center } & \multicolumn{3}{|c|}{ Validation center } \\
\hline & Presence [years] & Absence [years] & $P$-Value & Presence (years) & Absence (years] & $P$-Value \\
\hline & & & & & & \\
\hline Mean & 25.96 & 43.31 & $<0.0001$ & 29.43 & 37.65 & 0.026 \\
\hline SD & 14.525 & 12.321 & & 14.315 & 15.120 & \\
\hline \multicolumn{7}{|l|}{ 1st finger left } \\
\hline Mean & 27.00 & 44.68 & $<0.0001$ & 31.84 & 36.18 & 0.193 \\
\hline SD & 14.331 & 13.378 & & 15.771 & 14.640 & \\
\hline \multicolumn{7}{|l|}{ 5th finger right } \\
\hline Mean & 26.16 & 43.78 & $<0.0001$ & 29.88 & 35.49 & 0.223 \\
\hline SD & 14.754 & 11.485 & & 15.213 & 15.130 & \\
\hline \multicolumn{7}{|l|}{ 5th finger left } \\
\hline Mean & 27.89 & 43.54 & $<0.0001$ & 30.88 & 36.26 & 0.166 \\
\hline SD & 15.291 & 12.182 & & 12.959 & 16.377 & \\
\hline \multicolumn{7}{|l|}{ Elbow right } \\
\hline Mean & 24.33 & 35.05 & $<0.0001$ & 34.50 & 33.56 & 0.826 \\
\hline SD & 14.069 & 15.802 & & 16.688 & 13.189 & \\
\hline \multicolumn{7}{|l|}{ Elbow left } \\
\hline Mean & 26.33 & 36.19 & 0.001 & 30.97 & 37.77 & 0.1 \\
\hline SD & 14.686 & 15.835 & & 16.043 & 13.574 & \\
\hline \multicolumn{7}{|l|}{ Knee right } \\
\hline Mean & 28.17 & 35.77 & 0.015 & 26.94 & 36.85 & 0.032 \\
\hline SD & 16.753 & 13.957 & & 14.627 & 14.690 & \\
\hline \multicolumn{7}{|l|}{ Knee left } \\
\hline Mean & 28.19 & 36.04 & 0.009 & 27.64 & 37.42 & 0.016 \\
\hline SD & 16.360 & 14.429 & & 14.549 & 14.641 & \\
\hline \multicolumn{7}{|l|}{ Spine } \\
\hline Mean & 34.80 & 29.39 & 0.048 & 31.84 & 37.11 & 0.107 \\
\hline SD & 14.502 & 16.545 & & 15.833 & 14.106 & \\
\hline \multicolumn{7}{|c|}{$\begin{array}{l}\text { Significant } P \text {-values below } 0.01 \text { are indicated in bold. } P \text {-values were calculated with the Mann-Whitney } U \text { test. } \\
\text { Mean and standard deviation are expressed in years. } \\
\text { SD, standard deviation. }\end{array}$} \\
\hline
\end{tabular}

were noted in males for an excess of hypermobile first $(P$-value: $0.04)$ and fifth ( $P$-value: 0.01$)$ fingers on the right in the TC only. All other BS items were comparable between sexes in both groups. A mean BS value higher in males (6.5) was registered in the TC only. Table IV showed comparison of the other structural features (hypermobility at extra joints, anthropometry and orthopedic traits) between sexes. No differences were noted on anthropometry and only a few significant divergences were registered for the other features. Males resulted more commonly hypermobile than females at neck flexion (both sides), while females showed more frequently cubitus valgus on the left.

\section{Joint Instability Complications}

In order to test whether age at ascertainment might relate with presence of joint instability complications (i.e., dislocations, sprains, and soft-tissue injuries) at history taking, patients were arbitrarily stratified by age (i.e., $\leq 20$ years, $21-30$ years, $31-40$ years, $>40$ years). Sites (dislocations and sprains only) and rate of joint instability complications was evaluated accordingly (Table V). No major significant difference was registered in term of positive report of previous dislocations and sprains among age categories in both groups. Comparable results were also obtained for sites of dislocations and sprains. A significantly higher rate of soft tissue injuries (three or more events) was registered in adults and elder of the TC only. Tendon and muscle ruptures had a very low rate in both groups and, then, may be considered occasional findings.

\section{Handedness}

Patients from the TC were also investigated for a possible role of handedness on the rate of joint hypermobility and bilateral orthopedic traits. No significant difference was observed for fixed subluxation of the distal ulnar epyphysis and 1st metacarpal, genuum valgus, hallux valgus, cubitus valgus, in-toeing gait, flexible flatfoot, and joint hypermobility at knee, elbow, 1st and 5th fingers, 2nd-4th distal and proximal interphalangeal joint of hands, metacarpophalangeal joint, spine (rotation and flexion), neck (rotation and flexion), 1st metatarsophalangeal joint, ankles, and hips. 

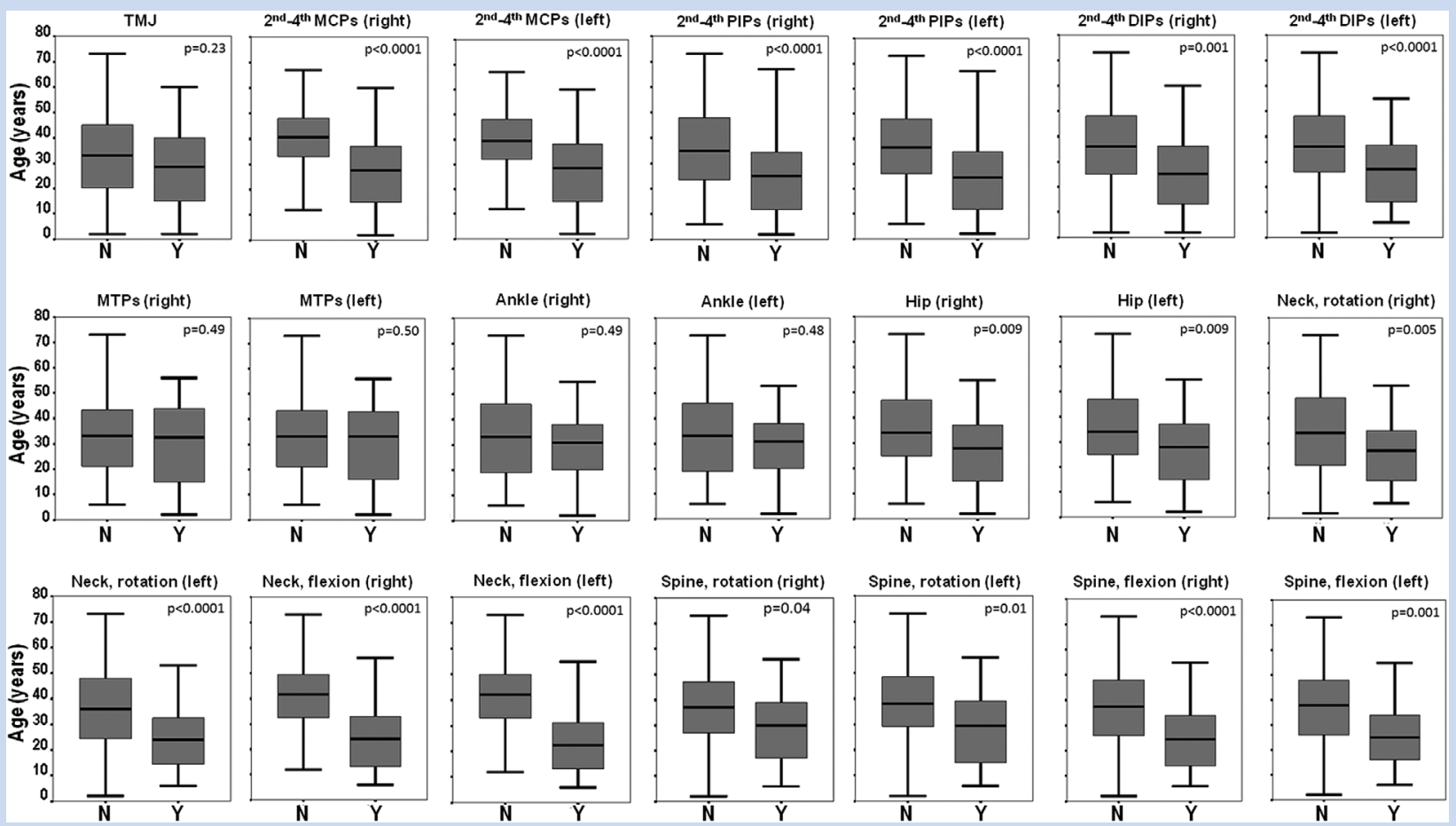

FIG. 2. Box plots comparing the presence $[Y=y e s]$ and absence $(N=n 0)$ of joint hypermobility [i.e., an excessive range of motion] at 21 joints or groups of joints outside the Beighton score in relation with age at examination. The horizontal black line indicates the median value, the upper box the first quartile, the lower box the remaining three quartiles, and the vertical line the entire range of values observed in the patient sample.

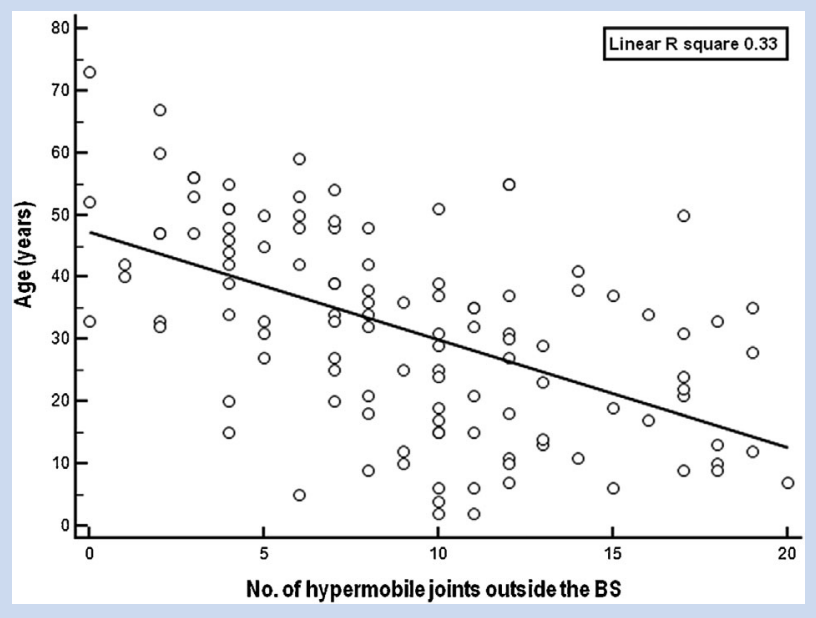

FIG. 3. Scatter plot showing inverse linear correlation between the presence of joint hypermobility at joints or groups of joints (no. 21) outside the Beighton score and age at examination of patients from the TC. For all joints, we attributed 1 point to their presence and 0 points to their absence for a maximum total of 21 .

\section{Multiple Correspondence Analysis and Multivariate Logistic Regression Analysis}

MCA for sex, BS $(\geq 5$ vs. $<5)$ and eleven selected orthopedic, and anthropometric traits did not depict a clear-cut clustering of features in discrete physical subphenotypes (Fig. 4). Anyway, low BS, brachymelia, and absence of the selected features (white dots) tend to associate together with a net separation from high BS and presence of orthopedic traits, dolichostenomelia according to the Brighton criteria (arm span/height ratio >1.03) and arachnodactyly (positive wrist or Steinberg signs - the clinical definition of arachnodactyly in JHS/ED-HT is a matter of debate and it will be probably included in the future diagnostic criteria). Male sex, pectus deformities, positive wrist sign, and flexible flatfoot are the most divergent features. More specifically, positive wrist sign tends to cluster with positive thumb sign, recurrent enthesopathies, and hallux valgus; pectus deformities segregate with male sex and associate with dolichostenomelia; and flexible flatfoot seems to combine with brachymelia and low BS.

Statistical analyses carried out with an univariate approach was further investigated with a multivariate logistic regression analysis. Positive results (i.e., with a $P$-value $<0.01$ ) were highlighted in Table VI. Significant influence of sex and evaluation center was also evident at the multivariate logistic regression analysis. Occasional significance was also registered for single orthopedic traits and joint instability complications. 
TABLE IV. Differences Between Sexes Within the Two Study Groups-Joint Hypermobility at Other Joints, Habitus and Orthopedic Traits

Training center $[\%]$

\section{Feature}

Joint hypermobility, other sites

TMJ

2nd-4th MCPs right

2nd-4th MCPs left

2nd-4th PIPs right

2nd-4th PIPs left

2nd-4th DIPs right

2nd-4th DIPs left

MTPs right

MTPs left

Ankle right

Ankle left

Hip right

Hip left

Neck rotation right

Neck rotation left

Neck flexion right

Neck flexion left

Full spine rotation right

Full spine rotation left

Full spine flexion right

Full spine rotation left

Total

Habitus (BMI)

Leptosomic

Picnic

Total

Habitus (arm span)

Dolichostenomelia

Brachymelia

Total

Wrist sign

Right

Left

Monolateral

Bilateral

Total

Thumb sign

Right

Left

Monolateral

Bilateral

Total

Orthopedic traits

FDSDU right

FDSDU left

1st MCP subluxation right

1st MCP subluxation left

Pectus carinatum

Pectus excavatum

Dorsal hyperkyphosis

Lumbar hyperlordosis

Scoliosis $(<20$ degrees $)$

Cubitus valgus right

Cubitus valgus left

\begin{tabular}{|c|c|c|}
\hline Females & Males & $P$-Value \\
\hline 38 (35.8) & $8(44.4)$ & 0.48 \\
\hline 68 (64.2) & 16 (88.9) & 0.04 \\
\hline 71 (67.0) & 16 (88.9) & 0.06 \\
\hline 32 (30.2) & 8 (44.4) & 0.23 \\
\hline 42 (39.6) & $8(44.4)$ & 0.70 \\
\hline 49 (46.2) & $9(50.0)$ & 0.77 \\
\hline 52 (49.1) & 9 (50.0) & 0.94 \\
\hline 40 (40.4) & $11(61.1)$ & 0.10 \\
\hline $40[40.4]$ & 12 (66.7) & 0.04 \\
\hline 29 (29.3) & 3 (16.7) & 0.39 \\
\hline 29 (29.3) & 3 (16.7) & 0.39 \\
\hline $43(43.4)$ & 6 (33.3) & 0.42 \\
\hline 43 (43.4) & 6 (33.3) & 0.42 \\
\hline 33 (32.4) & 5 (33.3) & 0.99 \\
\hline 30 (29.4) & 5 (33.3) & 0.76 \\
\hline 45 (44.1) & $12(80.0)$ & 0.009 \\
\hline 44 (43.1] & $12(80.0)$ & 0.008 \\
\hline 65 (63.7) & 13 (86.7) & 0.08 \\
\hline 65 (63.7) & 13 (86.7) & 0.08 \\
\hline 34 (33.3) & 8 (53.3) & 0.13 \\
\hline 36 (35.3) & 8 (53.3) & 0.18 \\
\hline $15-18$ & $99-102$ & \\
\hline $14(15.6)$ & 1 (16.7) & 0.99 \\
\hline 22 (24.4) & 1 (16.7) & 0.99 \\
\hline 90 & 6 & \\
\hline 24 (23.3] & 6 (33.3) & 0.38 \\
\hline 11 (10.7) & $1(5.6)$ & 0.69 \\
\hline 103 & 18 & \\
\hline 24 (22.9) & $2(12.5)$ & 0.52 \\
\hline 24 (22.9) & $3(18.8)$ & 0.99 \\
\hline $4(3.8)$ & 1 (6.3) & 0.51 \\
\hline 22 (21.0) & $2(12.5)$ & 0.74 \\
\hline 105 & 16 & \\
\hline $10(9.5)$ & 1 (5.9) & 0.99 \\
\hline 14 (13.3) & $0(0.0)$ & 0.21 \\
\hline 4 (3.8) & $1(5.9)$ & 0.53 \\
\hline $10(9.5)$ & $0(0.0)$ & 0.35 \\
\hline 105 & 17 & \\
\hline 73 (69.5) & 8 (44.4) & 0.04 \\
\hline 76 (71.7) & 10 (55.6) & 0.17 \\
\hline 6 (5.7) & 1 (5.6) & 0.99 \\
\hline 4 (3.8) & $1(5.6)$ & 0.55 \\
\hline 3 (2.9) & 2 (11.1) & 0.11 \\
\hline $5(4.9)$ & 4 (22.2) & 0.03 \\
\hline 70 (66.0) & 13 (76.5) & 0.39 \\
\hline 75 [70.8) & 11 (61.1) & 0.41 \\
\hline 60 (56.6) & 7 (38.9) & 0.16 \\
\hline 60 (56.6) & $5(27.8)$ & 0.02 \\
\hline 51 (48.1) & $1(5.6)$ & 0.001 \\
\hline
\end{tabular}




Feature
Genuum valgus right
Genuum valgus left
Flexible flatfoot right
Flexible flatfoot left
In-toeing gait right
In-toeing gait left
Hallux valgus right
Hallux valgus left
Total

Feature

Genuum valgus left

Flexible flatfoot right

Flexible flatfoot left

Hallux valgus left

\section{Training center $[\%]$}

\begin{tabular}{c}
\hline Females \\
$52[49.1]$ \\
$54[50.9]$ \\
$58(54.7)$ \\
$54[50.9]$ \\
$6[5.7)$ \\
$6[5.7)$ \\
$32[30.5]$ \\
$33(31.4)$
\end{tabular}

105-106

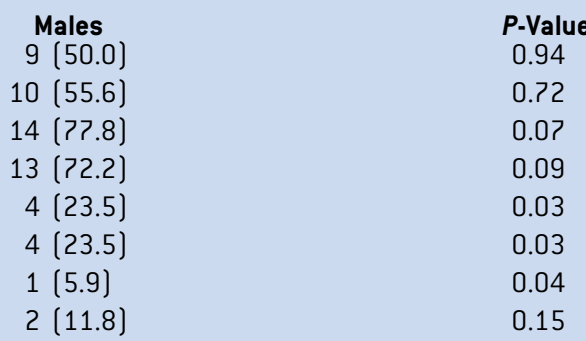

18

Significant $P$-values below 0.01 are indicated in bold

Comparison by sex was not possible in the validation center due to the small number of patients.

DIP, distal interphalangeal (joint); MCP, metacarpophalangeal (joint); MTP, metatarsophalangeal (joint); NA, not available; PIP, proximal interphalangeal (joint); TMJ, temporomandibular joint.

\section{DISCUSSION}

The leading purpose of this study is nosologic. Since the publication of the two currently available diagnostic criteria for JHS and EDS-HT in the late '90s [Beighton et al., 1998; Grahame et al., 2000], too little attention has been paid to the features detectable on physical examination. In fact, in the last 5 years the number of publications on JHS/EDS-HT has multiplied, but many of them were limited to analyzing subjective symptoms or variables influencing quality of life. However, thanks to the efforts of a few research groups, JHS/EDS-HT is now a complex and truly "systemic" disorder overlapping functional gastrointestinal disorders [Zarate et al., 2010] and cardiovascular dysautonomia [De Wandele et al., 2014]. Pain generation and modulation in JHS/EDS-HT is a further promising field of research in this condition [Rombaut et al., 2015b]. It is likely that JHS/EDS-HT probably will remain for years a clinical diagnosis recognized during consultation by accurate physical examination. Now, it is clear enough that there is a lack of consensus concerning the application of Brighton and Villefranche criteria and BS also among experts and such a confusion is influenced by the lack of clinical research on the physical phenotype and natural history of JHS/EDS-HT [Remvig et al., 2014; Castori et al., 2014b]. The phenotypic continuity from apparently isolated GJH on one end and JHS/EDS-HT on the other is difficulty managed also in specialized setting, and this lays on the gap between available diagnostic criteria and the increasing knowledge accumulated in the last years.

The inverse relationship between age at diagnosis and ROM and, consequently, BS is known since 2007 [Remvig et al., 2007]. In the present study, we confirmed that in Italian JHS/EDS-HT patients, the BS is lower in adults and is often in the "negative" range (i.e., $<5)$ since the fourth decade of life [Castori et al., 2011, 2014a, 2015]. This means that pediatric and young adult patients commonly have a "positive" BS at first ascertainment. Then, in the presence of the typical but variable skin changes, they can be labeled as EDS-HT according to Villefranche criteria. In the meantime, adults and the elder rarely maintain or obtain a formal diagnosis of EDS-HT due to their often low BS. Age was demonstrated as a primary confounding factor in the mutual application of Brighton and Villefranche criteria in a pedigree study on 23 families with
JHS/EDS-HT [Castori et al., 2014a]. In this work, most families showed a non-homogeneous distribution of the two clinical diagnoses among the different generations, with an excess of EDS-HT "alone" in children and JHS in adults and elders, and possibility of co-morbidity in adolescents and middle-aged patients.

The question whether a patient with the suspect of a systemic disorder has (had) or not GJH is relevant. In fact, the differential diagnosis of such "complex" patients is not straightforward and often include many rheumatologic and non-rheumatologic disorders. In the presence of GJH and in the absence of chronic inflammation, the diagnosis of JHS/EDS-HT is likely. The negative effect of age on ROM was known in the past [Grahame et al., 2000; Remvig et al., 2007]. Probably in line with such an assumption, the Brighton criteria, which were originally defined in an adult rheumatologic setting, include a lower BS $(\geq 4)$ compared to the Villefranche criteria $(\geq 5)$ as a major criterion and also consider a BS value of 1-3 as a minor criterion. In addition, a simple 5-point questionnaire was subsequently "unofficially" introduced in order to historically check for GJH in symptomatic or asymptomatic patients who have lost their earlier hypermobility [Hakim and Grahame, 2003].

In this study, we observed that age at diagnosis has some relationship with the single items of the BS, as well as with the ROM of 21 additional joints or group of joints. While the BS tends to be lower in adults and elder in both groups, the link between age at ascertainment and the presence/absence of the single BS items is different in the two patients' cohorts. In particular, the presence of seven out of the nine maneuvers of the BS associate with a lower mean age at diagnosis in the TC only. Furthermore, the mean BS is overall higher in the TC than VC. The reasons for such a difference remain obscure. Interestingly, while in the TC the BS was assessed by the use of an orthopedic goniometer, this was not the case of the VC where the evaluation of ROM was clinical. It may be inferred that the routine use of an orthopedic goniometer may contribute to this heterogeneity. Anyway, further elements, such as small sample size, different personnel/centers, lack of standardization and not comparable training background, could have influenced the divergent results on BS between the two centers. In addition, there are more differences between the TC and VC and no attempt was carried out to explore the inter-examiner variability. This study confirms the lack of a consensus in the assessment of the BS also 
党

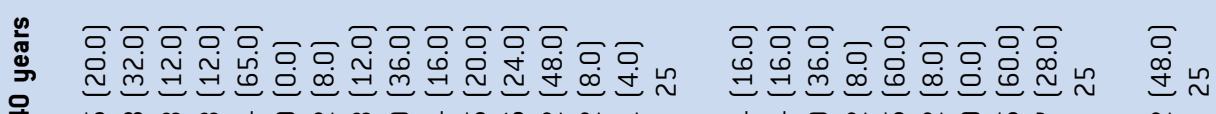

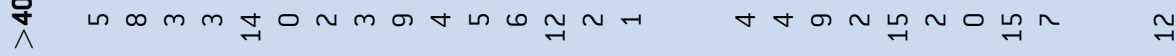

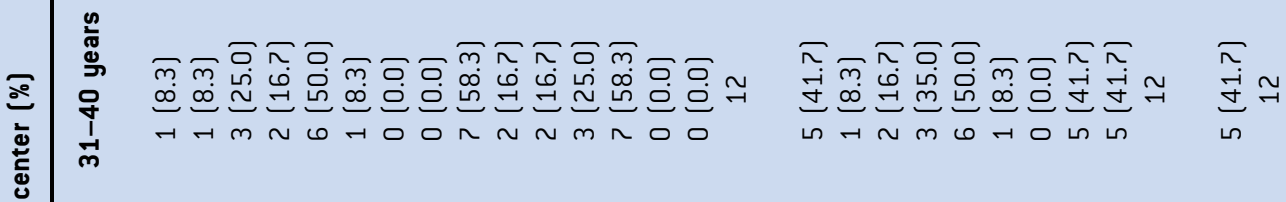

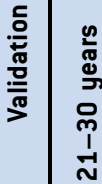

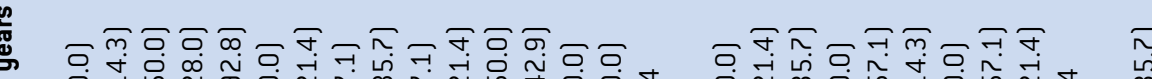

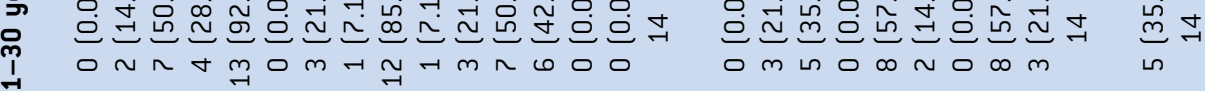

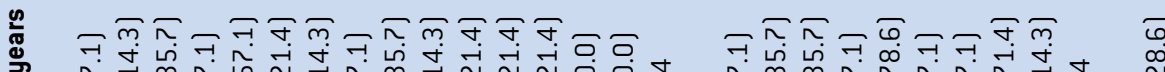

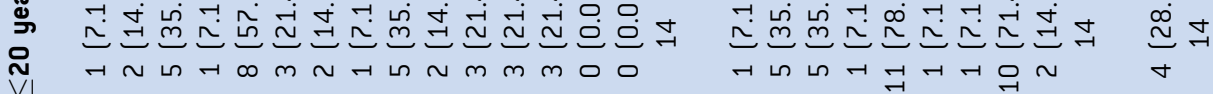

总

is

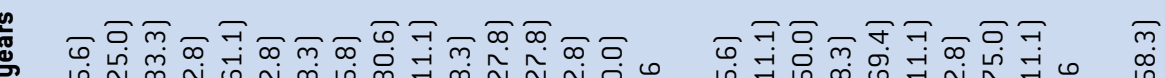

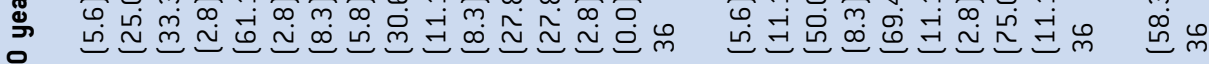

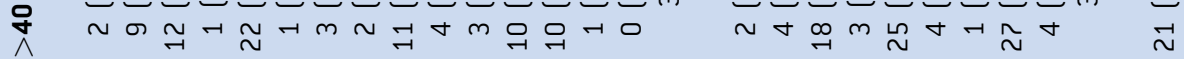

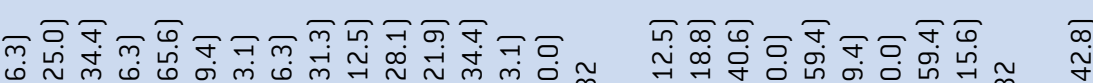

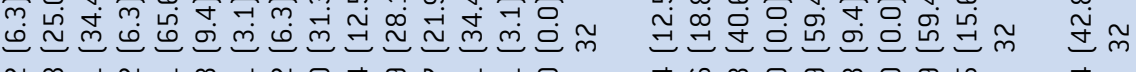

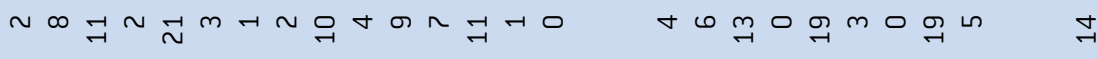

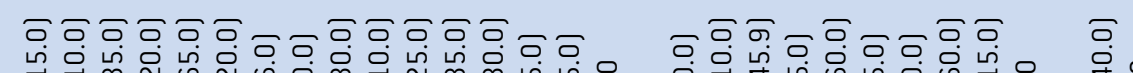

i m N

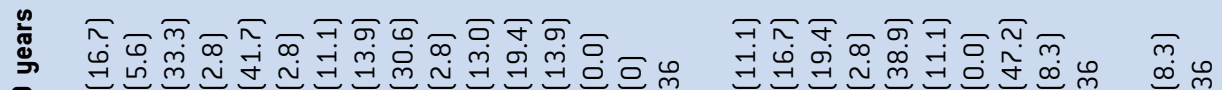

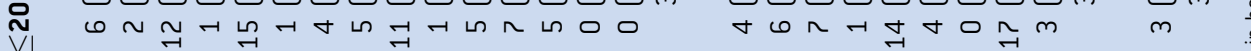




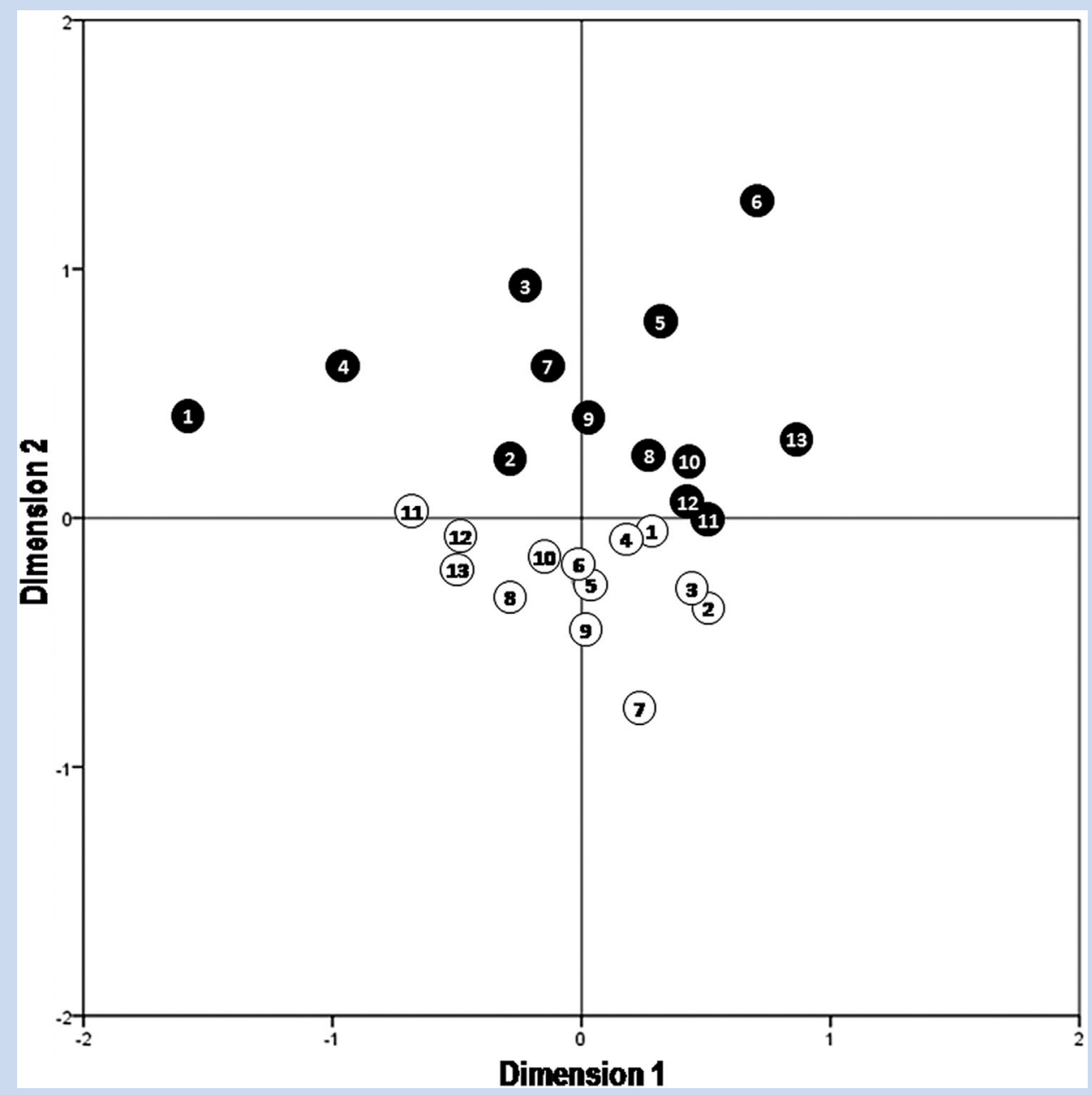

FIG. 4. Multiple correspondence analysis for sex, Beighton score [as a dichotomic trait; i.e., $\geq \mathbf{5}$ vs. $<5$ ] and eleven traits at physical examination. All features are indicated by a circle with black circles indicating presence and white circles indicating absence. This analysis was carried out in the training center only. Symbol legend: $\mathbf{1}=$ sex (black $=$ males; while $=$ females); $\mathbf{2}=$ Beighton score (black $=\geq \mathbf{5}$; white $=<5$ ); $3=$ arm span (black $>1.03$; white $=<0.9$ ) ; $4=$ sternum deformities (black $=$ yes; white $=$ no]; $5=$ thumb sign (black $=$ yes; white $=$ no); $6=$ wrist sign (black $=$ yes; white $=$ no); $7=$ flexible flatfoot (black $=$ yes; white $=$ no); $8=$ cubitus valgus $($ black $=$ yes; white $=$ no); $9=$ genuum valgus (black $=$ yes; white $=$ no); $10=$ hallux valgus (black $=$ yes; white $=$ no); $11=$ recurrent dislocations (black = yes; white $=$ no); $12=$ recurrent sprains (black $=$ yes; white $=$ no); $13=$ recurrent soft-tissue injuries (black $=$ yes; white $=$ no]. Dimension 1 and dimension 2 indicate the Euclidean spatial nature of the multiple correspondence analysis.

among "expert" centers [Remvig et al., 2014] and the need of more extended and multicentric studies for solving the issue.

A link between age at ascertainment and ROM of the 21 extra joints outside the BS is also evident. The mean age of patients showing hypermobility in many of these joints is significantly lower than the mean age of those with normal mobility at the same sites. This occurs in all joints but not in ankles, MTP joints, TMJ, and spine (in rotation), whose mobility, in turn, seemed less affected by age. However, such a phenomenon is lost at multivariate logistic regression analysis except for spine rotation on the left. Therefore, the search for joints less influenced by age in order to identify a more reliable scoring system for assessing GJH at different ages results prohibitive. In order to more accurately assess for the presence or absence of GJH, the routine evaluation of joints outside the BS may be helpful, especially when a full BS cannot be obtained due to non-collaborative or disabled patients, or in presence of a negative BS but a positive 5-point questionnaire.

Concerning joint instability complications, there is not any overt association between age at ascertainment and their rate and location. Multivariate logistic regression analysis demonstrated a link between age at diagnosis and presence of three or more soft tissue injuries only. Therefore, recurrent joint dislocations remain a valid marker for JHS/EDS-HT although a consensus is still lacking concerning number of dislocations and of affected joints for the 


\section{TABLE VI. Positive Results of the Multivariate Logistic Regression Analysis}

Sex

\section{Feature}

Habitus, picnic

Beighton score

1st finger right

1st finger left

5th finger right

5 th finger left

Elbow right

Elbow left

Knee right

Knee left

Spine

Hypermobility, other joints

2nd-4th DIPs right

2nd-4th DIPs left

2nd-4th PIPs right

2nd-4th PIPs left

2nd-4th MCPs right

2nd-4th MCPs left

Full spine rotation left

Full spine flexion right

Full spine flexion left

Neck rotation right

Neck rotation left

Neck flexion right

Neck flexion left

Hip right

Hip left

Orthopedic traits

FDSDU right

FDSDU left

1st MCP subluxation right

1st MCP subluxation left

Pectus excavatum

Lumbar hyperlordosis

Cubitus valgus right

Cubitus valgus left

Genuum valgus right

Genuum valgus left

In-toeing gait left

Hallux valgus right

Hallux valgus left

Dislocations

Number: $3-10$

Number: habitual/recurrent

Site: Shoulders

Site: TMJ

Sprains

Number: $>10$

Site: knees

Three or more soft tissue injuries

\section{OR (C195\%)}

OR [Cl 95\%]

$1.05(0.20-5.60)$

$0.97(0.30-3.10)$

$0.78(0.25-2.49)$

$1.74(0.51-5.99)$

$0.96(0.30-3.09)$

$1.51(0.60-3.87)$

$0.91(0.35-2.33)$

$0.89(0.33-2.36)$

$1.18(0.44-3.14)$

$0.40(0.15-1.06)$

$0.43(0.13-1.41)$

$0.34(0.10-1.16)$

$0.64(0.19-2.13)$

$0.41(0.12-1.37)$

$1.68(0.31-9.05)$

$1.60(0.30-8.50)$

$2.34(0.47-11.80)$

$1.01(0.29-3.50)$

$0.98(0.29-3.37)$

$0.46(0.12-1.69)$

$0.44(0.12-1.66)$

$1.77(0.38-8.33)$

$1.50(0.29-7.67)$

$0.23(0.07-0.82)$

$0.23(0.07-0.82)$

$0.81(0.25-2.62)$

$1.23(0.38-4.03)$

NA

NA

$5.83(1.77-19.27)$

$0.45(0.17-1.17)$

$0.32(0.12-0.84)$

$0.11(0.03-0.40)$

$0.84(0.34-2.09)$

$1.58(0.64-3.91)$

$2.62(0.75-9.17)$

$0.99(0.32-3.13)$

$1.25(0.41-3.76)$

$0.59(0.12-2.84)$

$1.21(0.29-5.05)$

$0.67(0.26-1.77)$

$0.67(0.22-2.03)$

$0.23(0.06-0.84)$

$2.62(0.83-8.26)$

$0.50(0.15-1.64)$
Age

\begin{tabular}{c}
\hline OR (CI 95\%) \\
$1.06(1.02-1.09)$
\end{tabular}

0.95

0.95

0.68

0.38

0.94

0.38

0.84

0.81

0.75

0.07

0.16

0.08

0.47

0.15

0.55

0.58

0.3

0.99

0.98

0.24

0.23

0.47

0.63

0.02

0.02

0.72

0.73

$<0.0001$

0.1

0.02

$<0.0001$

0.7

0.32

0.13

0.99

1.05

0.51

0.8

0.42

0.67

0.03

0.1

0.25
$0.94(0.91-0.96)$

$0.94(0.92-0.97)$

$0.94(0.92-0.97)$

$0.95(0.93-0.97)$

$0.98(0.96-0.99)$

$0.96(0.94-0.98)$

$0.96(0.94-0.99)$

$0.97(0.94-0.99)$

1.01 (0.98-1.02)

$0.95(0.92-0.98)$

$0.95(0.92-0.97)$

$0.94(0.91-0.97)$

$0.94(0.92-0.97)$

$0.94(0.92-0.97)$

$0.95(0.92-0.98)$

$0.97(0.94-1.0)$

$0.95(0.92-0.98)$

$0.95(0.93-0.98)$

$0.95(0.92-0.98)$

$0.94(0.91-0.98)$

$0.93(0.90-0.96$

$0.91(0.88-0.95)$

$0.95(0.92-0.98)$

0.95 (0.92-0.98)

$1.05(1.02-1.08)$

1.05 (1.02-1.09)

$1.21(1.08-1.36)$

$1.16(1.05-1.28)$

$0.96(0.92-1.01)$

$0.98(0.95-0.99)$

$0.99(0.98-1.02)$

$0.98(0.96-1.01)$

$0.99(0.98-1.02)$

1.01 (0.99-1.03)

$0.96(0.92-1.0)$

$1.05(1.02-1.07)$

$1.05(1.02-1.07)$

$1.04(1.01-1.06)$

$0.99(0.96-1.03)$

0.99 (0.98-1.02)

$1.02(1.01-1.05)$

1.01 (0.99-1.03)

$1.03(0.99-1.06)$

$1.05(1.02-1.07)$

\section{$P$ \\ 0.001}

$<0.0001$
$<\mathbf{0 . 0 0 0 1}$
$<\mathbf{0 . 0 0 0 1}$
$<\mathbf{0 . 0 0 0 1}$
0.03
$<\mathbf{0 . 0 0 0 1}$
$\mathbf{0 . 0 0 1}$
$\mathbf{0 . 0 0 1}$
0.98

$<0.0001$

$<0.0001$

$<0.0001$

$<0.0001$

$<0.0001$

0.001

0.05

0.002

0.003

0.003

0.001

$<0.0001$

$<0.0001$

0.001

0.001

0.002

0.001

0.001

0.003

0.06

0.03

0.66

0.13

0.67

0.43

0.05

$<0.0001$

$<0.0001$

\subsection{1}

0.67

0.68

0.04

0.31

0.06

$<0.0001$
Center

\begin{tabular}{cc}
\hline OR (CI 95\%) & $\boldsymbol{P}$ \\
$1.10(0.49-2.49)$ & 0.81 \\
& \\
$3.08(1.54-6.16)$ & $\mathbf{0 . 0 0 1}$ \\
$3.44(1.73-6.85)$ & $<\mathbf{0 . 0 0 0 1}$ \\
$9.36(4.27-20.51)$ & $<\mathbf{0 . 0 0 0 1}$ \\
$5.61(2.78-11.34)$ & $<\mathbf{0 . 0 0 0 1}$ \\
$0.32(0.17-0.61)$ & $\mathbf{0 . 0 0 1}$ \\
$0.71(0.38-1.34)$ & 0.29 \\
$3.46(1.77 k 6.76)$ & $<\mathbf{0 . 0 0 0 1}$ \\
$2.74(1.43-5.23)$ & $\mathbf{0 . 0 0 2}$ \\
$0.54(0.29-0.99)$ & 0.05
\end{tabular}

$\begin{array}{ll}\text { NA } & \text { NA } \\ \text { NA } & \text { NA }\end{array}$

NA

NA

NA

NA

NA

NA

NA

NA

NA

NA

NA

NA

NA

NA

NA

NA

NA

NA

NA

NA

NA

NA

NA

NA

NA

NA

NA

NA

NA

NA

$0.34(0.11-1.02) \quad 0.06$

$1.03(0.53-1.99) \quad 0.93$

$1.02(0.55-1.88) \quad 0.96$

$0.96(0.51-1.80) \quad 0.89$

$1.88(1.01-3.51) \quad 0.05$

$1.85(1.0-3.44) \quad 0.05$

$0.72(0.24-2.20) \quad 0.57$

$0.58(0.30-1.14) \quad 0.11$

$0.55(0.28-1.08)$

0.08

$0.88(0.40-1.93)$

$0.37(0.14-0.99)$

0.74

0.05

0.007

$0.43(0.23-0.79)$

0.02

0.36

0.02

0.95 
formal attribution of this feature. Sprains are common complications of GJH in Italian patients but the high rate in the general population limits their use as a diagnostic criterion. Based on our preliminary study, recurrent (i.e., $\geq 3$ ) soft-tissue injuries seems a valid marker in adults but might lack sensitivity in children.

According to the generally accepted skewed sex ratio in JHS/ EDS-HT [Castori et al., 2010b], females were 6-7 times more highly represented than males also in both study groups. Variable explanations to this phenomenon have been recently put forward, including an autosomal trait with sex-restricted modifying factors, a complex disorder influenced by sexual dimorphism, and genetic heterogeneity also including a X-linked dominant form [Castori et al., 2011, 2014a]. Apparently in contrast with what was previously thought about the predominance of affected females and its simplistic explanation relating to the different muscular build between sexes, in this study JHS/EDS-HT males presented GJH with a similar pattern compared to females (Table IV). This phenomenon remains without a clear explanation, but may be still in line with the tendency of an attenuated phenotype in males. Accordingly, in males symptoms may present less frequently and usually in those with more marked physical manifestations, such as a positive or higher BS. Besides perhaps a slightly significant increase of the mean BS in males, no other physical attribute seems appropriate for distinguishing between sexes.

Typical habitus was heterogeneously reported feature of our samples. The role of Marfanoid habitus was early recognized and included in the Brighton criteria [Grahame et al., 2000]. More recently, emphasis has been put on arachnodactyly as a powerful marker for heritable connective tissue disorders in the routine rheumatologic clinic [Grahame and Hakim, 2013]. Habitus is not included in the Villefranche criteria for EDS-HT and the original habitus item from the Brighton criteria does not offer any guidance on the relative weight that dolichostenomelia and arachnodactyly carry in the attribution of the criterion. In our samples, dolichostenomelia, positive wrist sign and positive thumb sign were reported in $25 \%, 20-24 \%$, and $10-12 \%$ of the patients. Interestingly, dolichostenomelia and arachnodactyly did not combine together in our sample (Fig. 4). Therefore, our finding seems suggesting independence of the two sub-features during the assessment of the habitus in JHS/EDS-HT. Anthropometry was also independent from the distribution of the subcutaneous fat and BMI. We feel that in the past confusion could had occurred in the distinction between a true Marfanoid habitus and a leptosomic built (i.e., $\mathrm{BMI}<19$ ). Therefore, accurate anthropometric information is necessary before the attribution of the habitus criterion and further consensus is needed concerning the minimal number of positive maneuvers requested for establishing the presence of arachnodactyly. The middle finger length/total hand length ratio could be a useful and more objective surrogate of the wrist and thumb signs but systematic studies are still lacking in JHS/EDS-HT.

In summary, this work highlights the need of applying some adjustments for age concerning the assessment of GJH with minor emphasis on sexual dimorphism; the existence of a very few joints within and outside the BS that show a comparable ROM at the different ages at ascertainment; the high diagnostic value of recurrent and multiple-site dislocations compared with other joint instability complications; and the heterogeneity of orthopedic manifestations with dolichostenomelia and arachnodactyly occuring independently. We hope that our findings could support the future revision of the criteria presently defining the overlapping phenotypes of JHS and EDS-HT.

\section{ACKNOWLEDGMENTS}

The authors thank Prof. Rodney Grahame, Division of Medicine at the University College of London, for having critically revised the manuscript. This project was actively supported by C.E.D.I. Onlus, the Italian Ehlers-Danlos syndrome patients' association.

\section{REFERENCES}

Beighton P, Solomon L, Soskolne CL. 1973. Articular mobility in an African population. Ann Rheum Dis 32:413-418.

Beighton P, De Paepe A, Steinmann B, Tsipouras P, Wenstrup RJ. 1998. Ehlers-Danlos syndromes: Revised nosology, villefranche, 1997. EhlersDanlos national foundation (USA) and Ehlers-Danlos support group (UK). Am J Med Genet 77:31-37.

Castori M, Colombi M. 2015. Generalized joint hypermobility, joint hypermobility syndrome and Ehlers-Danlos syndrome, hypermobility type. Am J Med Genet Part C Semin Med Genet 169C:1-5.

Castori M, Colombi M. 2016. De la génétique à la clinique, approche diagnostique et thérapeutique du syndrome d'Ehlers-Danlos en Italie. J Réadapt Méd 36:8-27.

Castori M, Camerota F, Celletti C, Danese C, Santilli V, Saraceni VM, Grammatico P. 2010a. Natural history and manifestations of the hypermobility type Ehlers-Danlos syndrome: A pilot study on 21 patients. Am J Med Genet Part A 152A:556-564.

Castori M, Camerota F, Celletti C, Grammatico P, Padua L. 2010b. EhlersDanlos syndrome hypermobility type and the excess of affected females: Possible mechanisms and perspectives. Am J Med Genet Part A 152A:2406-2408.

Castori M, Sperduti I, Celletti C, Camerota F, Grammatico P. 2011. Symptom and joint mobility progression in the joint hypermobility syndrome (Ehlers-Danlos syndrome, hypermobility type). Clin Exp Rheumatol 29:998-1005.

Castori M, Morlino S, Celletti C, Ghibellini G, Bruschini M, Grammatico P, Blundo C, Camerota F. 2013. Re-writing the natural history of pain and related symptoms in the joint hypermobility syndrome/ Ehlers-Danlos syndrome, hypermobility type. Am J Med Genet Part A 161A:2989-3004.

Castori M, Dordoni C, Valiante M, Sperduti I, Ritelli M, Morlino S, Chiarelli N, Celletti C, Venturini M, Camerota F, Calzavara-Pinton P, Grammatico P, Colombi M. 2014a. Nosology and inheritance pattern(s) of joint hypermobility syndrome and Ehlers-Danlos syndrome, hypermobility type: A study of intrafamilial and interfamilial variability in 23 Italian pedigrees. Am J Med Genet Part A 164A:3010-3020.

Castori M, Morlino S, Grammatico P. 2014b. Towards a re-thinking of the clinical significance of generalized joint hypermobility, joint hypermobiity syndrome, and Ehlers-Danlos syndrome, hypermobility type. Am J Med Genet Part A 164A:588-590.

Castori M, Dordoni C, Morlino S, Sperduti I, Ritelli M, Valiante M, Chiarelli N, Zanca A, Celletti C, Venturini M, Camerota F, CalzavaraPinton P, Grammatico P, Colombi M. 2015. Spectrum of mucocutane- 
ous manifestations in 277 patients with joint hypermobility syndrome/ Ehlers-Danlos syndrome, hypermobility type. Am J Med Genet Part C Semin Med Genet 169C:43-53.

Castori M. 2012. Ehlers-Danlos syndrome, hypermobility type: An underdiagnosed hereditary connective tissue disorder with mucocutaneous, articular, and systemic manifestations. ISRN Dermatol 2012:751768.

Clarkson HM. 2012. Musculoskeletal assessment: Joint motion and muscle testing, 3rd Edition. Philadelphia (US): Lippincott Williams \& Wilkins.

De Paepe A, Malfait F. 2012. The Ehlers-Danlos syndrome, a disorder with many faces. Clin Genet 82:1-11.

De Wandele I, Rombaut L, Leybaert L, Van de Borne P, De Backer T, Malfait F, De Paepe A, Calders P. 2014. Dysautonomia and its underlying mechanisms in the hypermobility type of Ehlers-Danlos syndrome. Semin Arthritis Rheum 44:93-100.

Gharbiya M, Moramarco A, Castori M, Parisi F, Celletti C, Marenco M, Mariani I, Grammatico P, Camerota F. 2012. Ocular features in joint hypermobility syndrome/Ehlers-Danlos syndrome hypermobility type: A clinical and in vivo confocal microscopy study. Am J Ophthalmol 154:593-600.

Grahame R, Hakim AJ. 2013. Arachnodactyly_A key to diagnosing heritable disorders of connective tissue. Nat Rev Rheumatol 9:358-364.

Grahame R, Bird HA, Child A. 2000. The revised (Brighton 1998) criteria for the diagnosis of benign joint hypermobility syndrome (BJHS). J Rheumatol 27:1777-1779.

Greenacre M. 1984. Theory and applications of correspondence analysis. London: Academic Press.

Hakim AJ, Grahame R. 2003. A simple questionnaire to detect hypermobility: An adjunct to the assessment of patients with diffuse musculoskeletal pain. Int J Clin Pract 57:163-166.

Kirk JA, Ansell BM, Bywaters EG. 1967. The hypermobility syndrome. Musculoskeletal complaints associated with generalized joint hypermobility. Ann Rheum Dis 26:419-425.

Lebart L, Morineau A, Warwick KM. 1984. Multivariate descriptive statistical analysis. New York: Wiley.

Loeys BL, Dietz HC, Braverman AC, Callewaert BL, De Backer J, Devereux RB, Hilhorst-Hofstee Y, Jondeau G, Faivre L, Milewicz DM, Pyeritz RE, Sponseller PD, Wordsworth P, De Paepe AM. 2010. The revised Ghent nosology for the Marfan syndrome. J Med Genet 47:476-485.

Mayer K, Kennerknecht I, Steinmann B. 2013. Clinical utility gene card for: Ehlers-Danlos syndrome types I-VII and variants-Update 2012. Eur J Hum Genet 21. DOI: 10.1038/ejhg.2012.162
Palmer S, Terry R, Rimes KA, Clark C, Simmonds J, Horwood J. 2016. Physiotherapy management of joint hypermobility syndrome-A focus group study of patient and health professional perspectives. Physiotherapy 102:93-102.

Remvig L, Jensen DV, Ward RC. 2007. Epidemiology of general joint hypermobility and basis for a proposed criteria for benign joint hypermobility syndrome: Review of the literature. J Rheumatol 34: 804-809.

Remvig L, Flycht L, Christensen KB, Juul-Kristensen B. 2014. Lack of consensus on tests and criteria for generalized joint hypermobility, Ehlers-Danlos syndrome: Hypermobile type and joint hypermobility syndrome. Am J Med Genet Part A 164A:591-596.

Ritelli M, Dordoni C, Venturini M, Chiarelli N, Quinzani S, Traversa M, Zoppi N, Vascellaro A, Wischmeijer A, Manfredini E, Garavelli L, Calzavara-Pinton P, Colombi M. 2013. Clinical and molecular characterization of 40 patients with classic Ehlers-Danlos syndrome: Identification of 18 COL5A1 and 2 COL5A2 novel mutations. Orphanet J Rare Dis 8:58.

Rombaut L, Deane J, Simmonds J, De Wandele I, De Paepe A, Malfait F, Calders P. 2015a. Knowledge, assessment, and management of adults with joint hypermobility syndrome/Ehlers-Danlos syndrome hypermobility type among Flemish physiotherapists. Am J Med Genet Part C Semin Med Genet 169C:76-83.

Rombaut L, Scheper M, De Wandele I, De Vries J, Meeus M, Malfait F, Engelbert R, Calders P. 2015b. Chronic pain in patients with the hypermobility type of Ehlers-Danlos syndrome: Evidence for generalized hyperalgesia. Clin Rheumatol 34:1121-1129.

Tinkle BT, Bird HA, Grahame R, Lavallee M, Levy HP, Sillence D. 2009. The lack of clinical distinction between the hypermobility type of Ehlers-Danlos syndrome and the joint hypermobility syndrome (a.k.a. hypermobility syndrome). Am J Med Genet Part A 149A:2368-23670.

Voermans NC, Knoop H. 2011. Both pain and fatigue are important possible determinants of disability in patients with the Ehlers-Danlos syndrome hypermobility type. Disabil Rehabil 33:706-707.

Voermans NC, Knoop H, Bleijenberg G, van Engelen BG. 2010a. Pain in ehlers-danlos syndrome is common, severe, and associated with functional impairment. J Pain Symptom Manage 40:370-378.

Voermans NC, Knoop H, van de Kamp N, Hamel BC, Bleijenberg G, van Engelen BG. 2010b. Fatigue is a frequent and clinically relevant problem in Ehlers-Danlos Syndrome. Semin Arthritis Rheum 40:267-274.

Zarate N, Farmer AD, Grahame R, Mohammed SD, Knowles CH, Scott SM, Aziz Q. 2010. Unexplained gastrointestinal symptoms and joint hypermobility: Is connective tissue the missing link? Neurogastroenterol Motil 22:252-e78. 